TRANSACTIONS OF THE

AMERICAN MATHEMATICAL SOCIETY

Volume 363, Number 6, June 2011, Pages 2803-2820

S 0002-9947(2011)04917-2

Article electronically published on January 20, 2011

\title{
SEMI-TERMINAL CONTINUA IN KELLEY SPACES
}

\author{
JANUSZ R. PRAJS
}

\begin{abstract}
A continuum $K$ in a space $X$ is said to be semi-terminal if at least one out of every two disjoint continua in $X$ intersecting $K$ is contained in $K$. Based on this concept, new structural results on Kelley continua are obtained. In particular, two decomposition theorems for Kelley continua are presented. One of these theorems is an improved version of the aposyndetic decomposition theorem for Kelley continua.
\end{abstract}

The concept of a semi-terminal continuum, which is introduced in this article, captures and generalizes commonly known examples. If a continuum has an outlet point in the sense of [13], then it is semi-terminal. There are an abundance of semi-terminal continua with outlet points in spaces such as arcs, trees, dendrites and dendroids. If every point of a continuum is its outlet point, the continuum is terminal. Thus semi-terminality generalizes terminality of continua. Terminal continua proved to be important in the study of hereditarily indecomposable continua and homogeneous continua. Indeed, hereditary indecomposability can be defined as having only terminal subcontinua, and there are hereditarily indecomposable continua in all dimensions [1. Jones' aposyndetic decomposition theorem 11] asserts that nonaposyndetic homogeneous continua admit a decomposition into nondegenerate, terminal subcontinua with an aposyndetic quotient.

There exist semi-terminal continua, even in homogeneous spaces, having no outlet points. A class of such examples has been defined in a recent study [18] of the mutually aposyndetic decomposition, and reproduced below (Example 2.2) for the convenience of the reader. Semi-terminal continua in homogeneous spaces are a subject for another study. Here our approach is more general; we investigate semi-terminal continua in Kelley spaces.

The significance of the class of Kelley continua was noticed in the 1940s [12]. Since then, numerous results have been obtained showing that the division of all continua into those with regular properties, Kelley continua, and the others, nonKelley continua, is fundamental. Though there exist intriguing, important nonKelley continua, such as the Lelek fan [8] 14, most continua of interest tend to be Kelley. All locally connected continua are Kelley. On the other end of the spectrum, all hereditarily indecomposable continua are Kelley. So are all homogeneous continua. All absolute retracts for tree-like continua, and more generally, for hereditarily unicoherent continua, are also Kelley.

Received by the editors March 7, 2008.

2000 Mathematics Subject Classification. Primary 54F15; Secondary 54F50.

Key words and phrases. Ample, continuum, filament, homogeneous, semi-indecomposable, semi-terminal.

(C)2011 American Mathematical Society Reverts to public domain 28 years from publication 
To show that the theory developed here applies to wide collections of objects, we first introduce basic ideas and provide some classes of semi-terminal and semiindecomposable continua (section 2). Then we collect a number of general results showing regular properties of semi-terminal continua in all continua (section 3) and in Kelley continua (section 4). The structural results are included in sections 4 and 5.

Besides having abundance of significant examples of semi-terminal and semiindecomposable continua, two striking phenomena motivated the study presented in this article. First, our theory reveals a lot of new structural information in such general spaces as Kelley continua. Second, we find a deep analogy between the two following pairs of concepts for continua in Kelley spaces: (a) terminality and indecomposability; and (b) semi-terminality and semi-indecomposability.

\section{Preliminaries}

All spaces are assumed to be metric. A continuum is a compact, connected, nonempty space. A one-dimensional continuum is called a curve. The symbol $\operatorname{Lim} K_{n}$ stands for the limit of the sets $K_{n}$ in the sense of the Hausdorff distance. If $X$ is a space, the symbol $C(X)$ denotes the hyperspace of the subcontinua of $X$ equipped with the Hausdorff distance. A continuum is decomposable if it can be represented as the union of two of its proper subcontinua. Otherwise it is called indecomposable. It is easy to see that a continuum $X$ is indecomposable if and only if $X$ is the only subcontinuum of $X$ with nonempty interior. A hereditarily indecomposable continuum is a continuum whose every subcontinuum is indecomposable. A subcontinuum $T$ of a space $X$ is called terminal in $X$ provided that for every subcontinuum $Y$ of $X$ that intersects $T$, either $Y \subset T$ or $T \subset Y$. Note that a continuum $X$ is hereditarily indecomposable if and only if every subcontinuum of $X$ is terminal. A point $p$ in a subcontinuum $Y$ of a space $X$ is called an outlet point of $Y$ provided that $p \in Z$ for every continuum $Z$ in $X$ such that $Z \cap Y \neq \emptyset \neq Z-Y$. We observe that a subcontinuum $T$ of a space $X$ is terminal if and only if every point of $T$ is its outlet point.

All maps are assumed to be continuous. A map $f: X \rightarrow Y$ between spaces $X$ and $Y$ is called monotone if $f^{-1}(p)$ is connected for every $p \in Y$.

A space $X$ is called a Kelley space if, whenever a sequence $\left\{x_{n}\right\} \subset X$ converges to a point $x \in X$ and $x$ belongs to a subcontinuum $K$ of $X$, there are continua $K_{n}$ such that $x_{n} \in K_{n}$ and $\operatorname{Lim} K_{n}=K$ [12. A space $X$ is said to be homogeneous provided that for every $x, y \in X$ there exists a homeomorphism $h: X \rightarrow X$ such that $h(x)=y$. Every homogeneous continuum is a Kelley space 30.

A space $X$ is said to be aposyndetic at $x$ with respect to $y$ if there exists a continuum $K$ in $X$ such that $x \in \operatorname{Int} K$ and $y \notin K$. If $X$ is aposyndetic at $x$ with respect to $y$ for every two different points $x$ and $y$ in $X$, then $X$ is called aposyndetic. If for every two different points $x, y \in X$ there are disjoint continua $K_{x}$ and $K_{y}$ such that $x \in \operatorname{Int} K_{x}$ and $y \in \operatorname{Int} K_{y}$, then $X$ is called mutually aposyndetic.

The following concepts have been introduced in [21] and further developed in [18], [19, 20], 22], [23], and 24]. If $X$ is a space, a subcontinuum $K$ is called filament (in $X$ ) provided there exists a neighborhood $N$ of $K$ such that the component of $N$ containing $K$ has empty interior. If $X$ is a continuum, $p \in X$, and every subcontinuum of $X-\{p\}$ has empty interior, then $p$ is called a cofilament point of $X$ (see 20, for a more systematic study of cofilament sets). Note that $p$ is a 
cofilament point of $X$ if and only if every subcontinuum of $X-\{p\}$ is filament, and, $X$ is indecomposable if and only if each $p \in X$ is a cofilament point of $X$. A subcontinuum $A$ of $X$ is called ample if for every neighborhood $U$ of $A$ there is a continuum $B$ such that $A \subset \operatorname{Int} B \subset B \subset U$. Proposition 1.1 below, which has been proved in [21, Proposition 2.3], is fundamental to the study of filament and ample continua in Kelley spaces. By this proposition, the set of cofilament points in a Kelley continuum is the intersection of all ample subcontinua of the space. More generally, in the study of concepts such as aposyndesis, mutual aposyndesis, or semi-indecomposability (see section 2) in Kelley spaces, ample continua can often replace subcontinua with interiors.

Proposition 1.1. Every subcontinuum of a Kelley continuum $X$ is either filament or ample. In particular, if a continuum $Y$ in $X$ contains a nonfilament subcontinuum of $X$, then $Y$ is ample in $X$.

\section{Two CRUCIAL CONCEPTS AND SOME EXAMPLES}

The first of the two crucial concepts of this article is semi-indecomposability, which is an intrinsic topological property of a continuum. A continuum $X$ is said to be semi-indecomposable provided each two subcontinua of $X$ with interiors have nonempty intersection. Originally, this concept was introduced by Charles Hagopian in 1969 [9] under a different name of a strictly non-mutually aposyndetic continuum. Observe that a continuum $X$ is semi-indecomposable if and only if out of every two disjoint subcontinua of $X$ at least one is filament. Moreover, $X$ is indecomposable if and only if each two disjoint subcontinua of $X$ are filament. Thus semi-indecomposability is, in a sense, "half" or "semi" indecomposability.

If $X$ is a Kelley continuum, then $X$ is semi-indecomposable if and only if each two ample subcontinua of $X$ have nonempty intersection (see Proposition 1.1). All examples discussed in this section are Kelley continua. Clearly, a continuum having a cofilament point is semi-indecomposable. The Cantor fan, which is defined as the cone Cone $(\mathcal{C})$ over the Cantor set $\mathcal{C}$, seems to be the simplest nondegenerate, semiindecomposable continuum. Indeed, $\operatorname{Cone}(\mathcal{C})$ is semi-indecomposable because the vertex of $\operatorname{Cone}(\mathcal{C})$ is its cofilament point. It is also a hereditarily decomposable continuum.

Semi-indecomposability naturally occurs in the study of absolute retracts for hereditarily unicoherent continua (abbr. $A R(\mathcal{H U})$ ) and tree-like continua (abbr. $A R(\mathcal{T} \mathcal{L})$ ) [3, 4], [5, 6], 7], though the name semi-indecomposable is not used in these papers. It can be shown that each such absolute retract is either a Kelley dendroid or a non-path connected, semi-indecomposable continuum. Some of these absolute retracts, for instance the Cantor fan, are both path connected and semiindecomposable. It can be shown that if $X \in A R(\mathcal{H U})$ or $X \in A R(\mathcal{T} \mathcal{L})$, then $X$ is semi-indecomposable if and only if $X$ has some cofilament point. If $X$ is not path connected, the set of cofilament points coincides with the kernel of $X$ introduced in [3]. Since every tree-like continuum can be such a kernel of an $A R(\mathcal{T} \mathcal{L}),[3$, [5], the class $A R(\mathcal{T L})$ contains a large collection of decomposable, semi-indecomposable continua.

There exist semi-indecomposable continua $X$ without cofilament points. Since being a cofilament point is a topological property, in homogeneous continua either all or none of the points are cofilament. In [9, Theorem 10, p. 621] Hagopian has shown that the product $P \times P$ of the pseudo-arc $P$ with itself is semi-indecomposable. 
In a recent paper [19] the author has shown that if $S$ is a solenoid, then $S \times S$ is semi-indecomposable. Actually, a precise characterization of the pairs of solenoids whose products are semi-indecomposable is presented in this last article. The products $P \times P$ and $S \times S$ are homogenous, decomposable continua. Thus not every point of these spaces is cofilament. Hence $P \times P$ and $S \times S$ have no cofilament points.

Question 1. Does every decomposable, semi-indecomposable homogeneous continuum have dimension greater than 1 ?

The second crucial concept of this article is semi-terminality, which refers to the position of a continuum in a larger space.

Definition 2.1. A subcontinuum $Y$ of a space $X$ is called semi-terminal, provided that for each two disjoint subcontinua $Z_{1}$ and $Z_{2}$ of $X$ such that $Z_{1} \cap Y \neq \emptyset \neq Z_{2} \cap Y$, either $Z_{1} \subset Y$ or $Z_{2} \subset Y$.

Note that a subcontinuum $Y$ of a space $X$ is terminal if and only if for each two disjoint subcontinua $Z_{1}$ and $Z_{2}$ of $X$ such that $Z_{1} \cap Y \neq \emptyset \neq Z_{2} \cap Y$, both $Z_{1}$ and $Z_{2}$ are contained in $Y$. Thus semi-terminality indeed can be viewed as "half" or "semi" terminality.

In many cases semi-terminality of a subcontinuum is related to having an outlet point. Indeed, it is an immediate observation that a continuum having an outlet point is semi-terminal. As is mentioned in the introduction, an abundance of semiterminal continua with outlet points can be found in various classes of tree-like continua. In the study of aposyndesis all $L$-sets (see the definition in section 5) are semi-terminal subcontinua with outlet points. In particular, all members of the generalized Jones' aposyndetic decomposition of a Kelley continuum [23] are such continua.

Not all semi-terminal subcontinua have outlet points, even in homogeneous continua. The following examples have been introduced and studied in [18.

Example 2.2. Let $X$ be a homogeneous one-dimensional continuum, and let $\hat{X}$ be the continuous curve of pseudo-arcs with the quotient space $X$ and the quotient map $q: \hat{X} \rightarrow X$, as defined in [15. The fibers $q^{-1}(x)$ are terminal pseudo-arcs in $\hat{X}$. Define the diagonal $\Delta=\{(x, x) \mid x \in X\}$, and define $\hat{X}_{\Delta}^{2}=(q \times q)^{-1}(\Delta)$ as a subspace of $\hat{X} \times \hat{X}$. It is shown [18, Proposition 5.4] that any subcontinuum of $\hat{X}_{\Delta}^{2}$ that intersects a set $(q \times q)^{-1}(p)$ and its complement, for $p \in \Delta$, contains an ample subcontinuum of $(q \times q)^{-1}(p)$. On the other hand, $(q \times q)^{-1}(p)$ is the product $q^{-1}(p) \times q^{-1}(p)$ of two pseudo-arcs, and thus it is semi-indecomposable. Therefore, any two subcontinua intersecting $(q \times q)^{-1}(p)$ and its complement must intersect each other. Consequently, each continuum $(q \times q)^{-1}(p)$ is semi-terminal in $\hat{X}_{\Delta}^{2}$. By [18, Proposition 5.3], the group of self-homeomorphisms of $\hat{X}_{\Delta}^{2}$ that respect the decomposition into the continua $(q \times q)^{-1}(p)$ acts transitively on $\hat{X}_{\Delta}^{2}$. In particular, $\hat{X}_{\Delta}^{2}$ is homogeneous. Note that the continua $(q \times q)^{-1}(p)$ are not terminal in $\hat{X}_{\Delta}^{2}$. Indeed, the diagonal $\Delta_{0}=\{(x, x) \mid x \in \hat{X}\}$ of $\hat{X} \times \hat{X}$ is a continuum in $\hat{X}_{\Delta}^{2}$ intersecting each set $(q \times q)^{-1}(p)$ but containing none of them. Using the homeomorphisms that respect the decomposition of $\hat{X}_{\Delta}^{2}$ into the sets $(q \times q)^{-1}(p)$, we show that every point of $(q \times q)^{-1}(p)$ belongs to the complement of some continuum intersecting $(q \times q)^{-1}(p)$ and $\hat{X}_{\Delta}^{2}-(q \times q)^{-1}(p)$. Hence the sets $(q \times q)^{-1}(p)$ are semi-terminal subcontinua of $\hat{X}_{\Delta}^{2}$ without outlet points. 
Remark 2.3. If the continuum $X$ in Example 2.2 is mutually aposyndetic, the decomposition $\mathcal{Q}$ of $\hat{X}_{\Delta}^{2}$ into the continua $(q \times q)^{-1}(p)$ is the mutually aposyndetic decomposition of $\hat{X}_{\Delta}^{2}$ defined in [18. The properties of $\mathcal{Q}$ discussed in Example 2.2 may suggest, for homogeneous continua, a connection among the concepts of mutual aposyndesis, semi-indecomposability and semi-terminality, similar to the relation among aposyndesis, indecomposability and terminality known from Jones' aposyndetic decomposition theorem. This seems to be a promising idea for future research.

\section{Semi-terminal continua: General properties}

Proposition 3.1. If $Y$ and $Z$ are subcontinua of a space $X$ such that $Y$ is semiterminal, then $Y \cap Z$ is connected.

Proof. Suppose $C_{1}$ and $C_{2}$ are different components of $Y \cap Z$. Then there are continua $Z_{1}$ and $Z_{2}$ in $Z$, slightly larger than $C_{1}$ and $C_{2}$, respectively, such that $Z_{1} \cap Z_{2}=\emptyset$. We have $Z_{1}-Y \neq \emptyset \neq Z_{2}-Y$, which contradicts the semi-terminality of $Y$.

Corollary 3.2. Let $X$ be a continuum and $Y$ its semi-terminal subcontinuum. Then for every ample subcontinuum $A$ of $X$ the intersection $A \cap Y$ is an ample subcontinuum of $Y$.

Proof. Since $A$ is ample in $X$, it can be slightly enlarged to a continuum $B$ such that $A \subset \operatorname{Int} B$. By Proposition 3.1 the sets $A \cap Y$ and $B \cap Y$ are continua, and $A \cap Y$ is in the interior of $B \cap Y$ relative to $Y$. Clearly, if we take a sequence of such $B$ 's converging to $A$, then the sequence of their intersections with $Y$ converges to $A \cap Y$, and the needed conclusion follows.

Corollary 3.3. If $X$ is compact, $f: X \rightarrow Y$ is a monotone map, and $Z$ is a semiterminal subcontinuum of $X$, then the restriction $f \mid Z: Z \rightarrow Y$ is also monotone.

An easy proof of the following proposition is left to the reader.

Proposition 3.4. If $X$ is a compact space, $f: X \rightarrow Y$ is a monotone surjective map, and $Z$ is a semi-terminal subcontinuum of $X$, then $f(Z)$ is a semi-terminal subcontinuum of $Y$.

Corollary 3.5. Let $X$ be a compact space, $f: X \rightarrow Y$ a monotone surjective map, and $Z$ a subcontinuum of $X$ such that every subcontinuum of $Z$ is semi-terminal in $X$. Then every subcontinuum of $f(Z)$ is semi-terminal in $Y$.

Proof. Let $K$ be a subcontinuum of $f(Z)$. The restriction $f_{Z}=f \mid Z: Z \rightarrow f(Z)$ is monotone by Corollary 3.3. and thus $L=f_{Z}^{-1}(K)$ is a subcontinuum of $Z$ such that $f(L)=K$. By assumption, $L$ is semi-terminal in $X$. Thus $K=f(L)$ is semi-terminal in $Y$ by Proposition 3.4

Proposition 3.6. If $Y_{1}$ and $Y_{2}$ are semi-terminal subcontinua of a space $X$ and $Y_{1} \cap Y_{2} \neq \emptyset$, then $Y_{1} \cup Y_{2}$ is a semi-terminal subcontinuum of $X$.

Proof. If either $Y_{1} \subset Y_{2}$ or $Y_{2} \subset Y_{1}$, the conclusion is trivial. Assume $Y_{1}-Y_{2} \neq \emptyset \neq$ $Y_{2}-Y_{1}$. Suppose $Z_{1}$ and $Z_{2}$ are disjoint subcontinua of $X$ such that $Z_{1} \cap\left(Y_{1} \cup Y_{2}\right) \neq$ $\emptyset \neq Z_{2} \cap\left(Y_{1} \cup Y_{2}\right)$ and $Z_{1}-\left(Y_{1} \cup Y_{2}\right) \neq \emptyset \neq Z_{1}-\left(Y_{1} \cup Y_{2}\right)$. Then either $Z_{1} \cap Y_{1} \neq \emptyset$ or $Z_{1} \cap Y_{2} \neq \emptyset$. In the latter case, since $Y_{2}$ is semi-terminal, it follows that $Z_{1} \cap Y_{1} \neq \emptyset$. 
Thus $Z_{1} \cap Y_{1} \neq \emptyset$ in both cases. Similarly we argue that $Z_{2} \cap Y_{1} \neq \emptyset$. Since $Y_{1}$ is semi-terminal, either $Z_{1} \subset Y_{1} \subset Y_{1} \cup Y_{2}$ or $Z_{2} \subset Y_{1} \subset Y_{1} \cup Y_{2}$, a contradiction.

Definition 3.7. We say that a continuum $X$ is hereditarily semi-indecomposable whenever every subcontinuum of $X$ is semi-indecomposable.

Remark 3.8. Hereditarily semi-indecomposable continua are related to hereditarily indecomposable continua, which have been known and extensively studied since the 1920s. It is obvious that each hereditarily indecomposable continuum is hereditarily semi-indecomposable. The converse does not always hold. For instance, the one point union of two hereditarily indecomposable, nondegenerate continua (for instance, pseudo-arcs) is a decomposable, hereditarily semi-indecomposable continuum. In the next section it is shown (Corollary 4.10), however, that among Kelley continua the properties of being hereditarily semi-indecomposable and hereditarily indecomposable are equivalent.

It is known that for every continuum $X$ the collections of indecomposable and hereditarily indecomposable subcontinua of $X$ are $G_{\delta}$ subsets of $C(X)$. Here we observe the following.

Proposition 3.9. For every continuum $X$ the collections of semi-indecomposable and hereditarily semi-indecomposable subcontinua of $X$ are $G_{\delta}$ subsets of $C(X)$.

Proof. Let $d$ be a metric on $X$, and let $\mathcal{K}_{n}$ be the collection of subcontinua $K$ of $X$ such that $K$ contains subcontinua $L$ and $M$ satisfying: (i) $d(x, y) \geq 1 / n$ for all $x \in L$ and $y \in M$; and (ii) both $L$ and $M$ contain open balls relative to $K$ each of radius $1 / n$. We observe that $\mathcal{K}_{n}$ is a closed collection in $C(X)$, and the set of semi-indecomposable subcontinua of $X$ equals $C(X)-\bigcup_{n} \mathcal{K}_{n}$. The proof that the collections of hereditarily semi-indecomposable subcontinua of $X$ are a $G_{\delta}$ subset of $C(X)$ is left to the reader.

It is clear that a continuum is hereditarily indecomposable if and only if each of its subcontinua is terminal. Here we show a similar property for the hereditary semi-indecomposability and semi-terminality of continua.

Proposition 3.10. A continuum $X$ is hereditarily semi-indecomposable if and only if every subcontinuum of $X$ is semi-terminal in $X$.

Proof. Assume $X$ contains a non-semi-terminal subcontinuum $Y$. By definition there are disjoint continua $Z_{1}$ and $Z_{2}$ in $X$ such that $Z_{1} \cap Y \neq \emptyset \neq Z_{2} \cap Y$ and $Z_{1}-Y \neq \emptyset \neq Z_{2}-Y$. Thus $Z_{1}$ and $Z_{2}$ are disjoint subcontinua of the continuum $C=Z_{1} \cup Y \cup Z_{2} \subset X$ with interiors relative to $C$. Hence a subcontinuum $C$ of $X$ is not semi-indecomposable.

Assume $X$ contains a non-semi-indecomposable continuum $Y$. Throughout the remaining part of the proof all interiors are relative to $Y$. By definition there are disjoint subcontinua $Y_{0}$ and $Y_{1}$ of $Y$ each having nonempty interior. Let $p_{0}$ and $p_{1}$ be interior points of $Y_{0}$ and $Y_{1}$, respectively. Define a map $f: Y \rightarrow[0,1]$, letting

$$
f(y)=\frac{d\left(p_{0}, y\right)}{d\left(p_{0}, y\right)+d\left(y, p_{1}\right)},
$$

and note that $f$ is continuous, $f^{-1}(0)=\left\{p_{0}\right\}$ and $f^{-1}(1)=\left\{p_{1}\right\}$. Consequently, $f^{-1}\left(t_{0}\right) \subset \operatorname{Int} Y_{0}$ and $f^{-1}\left(t_{1}\right) \subset \operatorname{Int} Y_{1}$, for $t_{0}, t_{1} \in(0,1)$, whenever $t_{0}$ and $t_{1}$ are sufficiently near to 0 and 1 , respectively. Let $t_{0}$ and $t_{1}$ be such numbers. Since 
every map from a continuum onto $[0,1]$ is weakly confluent [16, Theorems 12.29 and 13.52], there exists a continuum $K \subset Y$ such that $f(K)=\left[t_{0}, t_{1}\right]$. Thus $K \cap Y_{0} \neq \emptyset \neq K \cap Y_{1}$, and neither $Y_{0}$ nor $Y_{1}$ is contained in $K$. Hence a subcontinuum $K$ of $X$ is not semi-terminal. The proof is complete.

\section{Semi-terminal continua in Kelley spaces}

Locally connected continua form an important and distinctive category within Kelley spaces. We begin this section with the following characterization of semiterminal subcontinua of locally connected continua.

Proposition 4.1. If $X$ is a locally connected continuum, then a proper subcontinuum $Y$ of $X$ is semi-terminal in $X$ if and only if $\operatorname{card}(\mathrm{bd} Y)=1$. In particular, each semi-terminal subcontinuum of $X$ is locally connected and has an outlet point.

Proof. Suppose $a, b \in \operatorname{bd} Y$ with $a \neq b$. Since $X$ is locally connected, there are disjoint continua $A$ and $B$ such that $a \in \operatorname{Int} A$ and $b \in \operatorname{Int} B$. We have $A \cap Y \neq$ $\emptyset \neq A-Y$ and $B \cap Y \neq \emptyset \neq B-Y$. Thus $Y$ is not semi-terminal, a contradiction. Therefore, the boundary of $Y$ is either a singleton or the empty set. It is easy to observe that if $p \in X$, then for every component $C$ of $X-\{p\}$ the closure $\operatorname{cl} C$ is a locally connected, semi-terminal subcontinuum of $X$. The proof is complete.

Proposition 4.2. Each semi-terminal subcontinuum of a Kelley continuum is a Kelley continuum.

Proof. Let $X$ be a Kelley continuum, and $Y$ be a semi-terminal subcontinuum of $X$. To prove that $Y$ is a Kelley continuum, take a continuum $K \subset Y$ and a sequence $\left\{p_{n}\right\} \subset Y$ converging to some point $p \in K$. Since $X$ is a Kelley continuum, there are continua $K_{n}^{\prime} \subset X$ such that $p_{n} \in K_{n}^{\prime}$ for each $n$, and $\operatorname{Lim} K_{n}^{\prime}=K$. Without loss of generality assume $K_{n}^{\prime}-Y \neq \emptyset$ for each $n$. By Proposition 3.1, the sets $L_{n}=K_{n}^{\prime} \cap Y$ are continua. Since $Y$ is semi-terminal, $K_{m}^{\prime} \cap K_{n}^{\prime} \neq \emptyset$ for each $m$ and $n$. Again by Proposition 3.1. the sets $L_{m} \cup L_{n}=\left(K_{m}^{\prime} \cup K_{n}^{\prime}\right) \cap Y$ are connected. Thus $L_{m} \cup L_{n}$ is a continuum for each $m$ and $n$. For fixed $n$, the sequence $P_{m}=L_{m} \cap L_{n}$ has some limit point in $K$, and thus $L_{n} \cap K \neq \emptyset$. Therefore $K_{n}=L_{n} \cup K$ is a subcontinuum of $Y$. Observe that $p_{n} \in K_{n} \subset Y$ for each $n$, and $\operatorname{Lim} K_{n}=K$, which completes the proof.

Proposition 4.3. If $Y$ is a semi-terminal subcontinuum of a Kelley continuum $X$, and $Z$ is a subcontinuum of $X$ such that $Z \cap Y \neq \emptyset \neq Z-Y$, then $Z \cap Y$ is an ample subcontinuum of $Y$.

Proof. Suppose $K=Z \cap Y$ is a filament subcontinuum of $Y$ and let $p \in K$. Since $K$ is filament, there is a neighborhood $U$ of $K$ in $Y$ such that the component $C$ of $U$ containing $K$ has empty interior relative to $Y$. Therefore, there is a sequence $\left\{p_{n}\right\} \subset U-C$ such that $\lim p_{n}=p$. Since $X$ is a Kelley continuum, there are continua $Z_{n}$ in $X$ converging to $Z$, in the sense of the Hausdorff distance, such that $p_{n} \in Z_{n}$. We have $Z-Y \neq \emptyset$, and thus $Z_{n}-Y \neq \emptyset$ for almost all $n$. Since $Y$ is semi-terminal, it follows that $Z_{n} \cap Z \neq \emptyset$ for almost all $n$. For sufficiently large $n$ we also have $Z_{n} \cap Y \subset U$. Thus the continuum $L=Z \cup Z_{n}$, for such $n$, satisfies $L \cap Y \subset U$ and $L \cap C \neq \emptyset \neq U-C$. Hence $L \cap K$ is not connected, which is impossible by Proposition 3.1 .

We have shown that $K$ is not filament in $Y$. Since $Y$ is a Kelley continuum by Proposition 4.2, the continuum $K$ is ample in $Y$ by Proposition 1.1. 
Corollary 4.4. Let $X$ be a Kelley continuum and $Y$ its semi-terminal subcontinuum. Then every cofilament point of $Y$ is an outlet point of $Y$. In particular, every indecomposable semi-terminal subcontinuum of a Kelley continuum $X$ is terminal in $X$.

Proof. If $p$ is not an outlet point of $Y$, then some continuum $Z \subset X$ satisfies $Z \cap Y \neq \emptyset \neq Z-Y$ and $p \notin Z$. The set $Z \cap Y$ is an ample subcontinuum of $Y$ by Proposition 4.3, and thus $p$ is not cofilament in $Y$.

If $Y$ is indecomposable, $p$ is cofilament for each $p \in Y$, and thus each $p \in Y$ is an outlet point of $Y$. Hence $Y$ is terminal in $X$.

It is known that the terminal subcontinua of a Kelley continuum form a closed collection in $C(X)$. Here we show a similar result for semi-terminal continua.

Proposition 4.5. If $X$ is a compact Kelley space, then the collection $\mathcal{S T}(X)$ of semi-terminal subcontinua of $X$ is closed in $C(X)$.

Proof. Let $K$ be a non-semi-terminal subcontinuum of $X$, and $K_{n}$ a sequence of subcontinua of $X$ converging to $K$ in the sense of the Hausdoff distance. Since $K$ is non-semi-terminal, there are disjoint continua $P$ and $Q$ such that $P \cap K \neq \emptyset \neq Q \cap K$ and $P-K \neq \emptyset \neq Q-K$. Let $p \in P \cap Y$ and $q \in Q \cap Y$. We have $\lim K_{n}=K$, and thus there exist $\left\{p_{n}\right\} \subset X$ and $\left\{q_{n}\right\} \subset X$ such that $p_{n}, q_{n} \in K_{n}, \lim p_{n}=p$ and $\lim q_{n}=q$. Since $X$ is a Kelley space, there are continua $P_{n}$ and $Q_{n}$ such that $p_{n} \in P_{n}, q_{n} \in Q_{n}, \lim P_{n}=P$, and $\lim Q_{n}=Q$. Since $P \cap Q=\emptyset$, it follows that $P_{n} \cap Q_{n}=\emptyset$ for almost all $n$. Similarly, since $P-K \neq \emptyset \neq Q-K$, we have $P_{n}-K_{n} \neq \emptyset \neq Q_{n}-K_{n}$ for almost all $n$. Thus almost all $K_{n}$ are non-semiterminal. Consequently, the collection of non-semi-terminal subcontinua of $X$ is open in $C(X)$. Hence $\mathcal{S} \mathcal{T}(X)$ is closed.

If $P$ is a subset of $X$ and $x \in X$, let $\mathcal{S T}(X, P, x)$ be the collection of semiterminal subcontinua $S$ of $X$ such that $x \in S \subset P$. Note that $\mathcal{S T}(X, P, x) \neq \emptyset$ if and only if $x \in P$.

Theorem 4.6. If $X$ is a compact Kelley space, $P$ is a closed subset of $X$ and $x \in P$, then the union $\Lambda(X, P, x)=\bigcup \mathcal{S} \mathcal{T}(X, P, x)$ is the largest member of $\mathcal{S} \mathcal{T}(X, P, x)$. Moreover, the collection $\Lambda(X, P)=\{\Lambda(X, P, x) \mid x \in P\}$ is an upper semi-decomposition of $P$ into the maximal semi-terminal subcontinua of $X$ contained in $P$.

Proof. Since $\mathcal{S T}(X)$ is closed in $C(X)$ by Proposition 4.5, so is $\mathcal{S T}(X, P, x)$. By Proposition 3.6 we have $Y_{1} \cup \cdots \cup Y_{n} \in \mathcal{S T}(X, P, x)$ whenever $Y_{1}, \ldots, Y_{n} \in$ $\mathcal{S T}(X, P, x)$. Finite unions of members of $\mathcal{S} \mathcal{T}(X, P, x)$ approximate $\Lambda(X, P, x)$, and thus $\Lambda(X, P, x)$ is the largest member of $\mathcal{S} \mathcal{T}(X, P, x)$. The continua $\Lambda(X, P, x)$, for varying $x$, are maximal semi-terminal subcontinua of $X$ in $P$ which are mutually disjoint by Proposition 3.6. Since the collection of semi-terminal subcontinua of $X$ contained in $P$ is closed in $C(X)$, the decomposition $\Lambda(X, P)$ is upper semicontinuous.

If $X$ is a space, let $\mathcal{H S T}(X)$ be the collection of hereditarily semi-terminal subcontinua of $X$, that is, the collection of the subcontinua $K$ of $X$ such that every subcontinuum of $K$ is semi-terminal in $X$.

Proposition 4.7. If $X$ is a Kelley continuum, then the collection $\mathcal{H S T}(X)$ of hereditarily semi-terminal subcontinua of $X$ is closed in $C(X)$. 
Proof. Suppose a sequence $\left\{K_{n}\right\} \subset \mathcal{H S T}(X)$ converges in $C(X)$ to a continuum $K \in C(X)-\mathcal{H S T}(X)$. Thus there exists a subcontinuum $L$ of $K$ that is not semi-terminal in $X$. Consequently, there exist disjoint continua $A$ and $B$ in $X$ such that $A \cap L \neq \emptyset \neq B \cap L$ and $A-L \neq \emptyset \neq B-L$. Let $a \in A \cap L, \quad b \in B \cap L$, and $\left\{a_{n}\right\} \subset X,\left\{b_{n}\right\} \subset X$ be sequences converging to $a$ and $b$, respectively, such that $a_{n}, b_{n} \in K_{n}$ for each $n$. Since $X$ is a Kelley continuum, there exist sequences $\left\{A_{n}\right\} \subset C(X)$ and $\left\{B_{n}\right\} \subset C(X)$ converging to $A$ and $B$, respectively, such that $a_{n} \in A_{n}$ and $b_{n} \in B_{n}$ for each $n$. Note that $A_{n} \cap B_{n}=\emptyset$ for almost all $n$. Similarly, there exist sequences $\left\{L_{a_{n}}\right\} \subset C(X)$ and $\left\{L_{b_{n}}\right\} \subset C(X)$, each converging to $L$, such that $a_{n} \in L_{a_{n}}$ and $b_{n} \in L_{b_{n}}$ for each $n$.

Case 1 . Either infinitely many $L_{a_{n}}$ 's or infinitely many $L_{b_{n}}$ 's are contained in the corresponding $K_{n}$ 's.

Since $K_{n} \in \mathcal{H S T}(X)$, every subcontinuum of $K_{n}$ is semi-terminal. In this case, $L$ is the limit semi-terminal continua, and thus it is semi-terminal by Proposition 4.5, a contradiction.

Case 2. $L_{a_{n}}-K_{n} \neq \emptyset \neq L_{b_{n}}-K_{n}$ for almost all $n$.

Without loss of generality, assume $L_{a_{n}}-K_{n} \neq \emptyset \neq L_{b_{n}}-K_{n}$ for each $n$. Since each $K_{n}$ is semi-terminal, it follows that $L_{a_{n}} \cap L_{b_{n}} \neq \emptyset$ for each $n$, and thus the sets $M_{n}=L_{a_{n}} \cup L_{b_{n}}$ are continua. By Proposition 3.1 the intersections $P_{n}=M_{n} \cap K_{n}$ are continua, which satisfy $a_{n}, b_{n} \in P_{n}$ for each $n$. Since the continua $M_{n}$ converge to $L$, we also have $A_{n}-P_{n} \neq \emptyset \neq B_{n}-P_{n}$ for almost all $n$. Hence almost all $K_{n}$ 's contain subcontinua, the corresponding $P_{n}$ 's, that are not semi-terminal. A contradiction.

Proposition 4.8. Each hereditarily semi-terminal subcontinuum of a Kelley continuum is indecomposable.

Proof. Suppose a decomposable subcontinuum $Y$ of a Kelley continuum $X$ is hereditarily semi-terminal. Then $Y$ is the union $K \cup L$ of two of its proper subcontinua $K$ and $L$. Since $Y$ is also hereditarily semi-terminal in itself, by Proposition 3.10 each subcontinuum of $Y$ is semi-indecomposable. By Proposition 4.2, the continuum $K$ is Kelley. By Proposition 4.3 the set $A=K \cap L$ is an ample subcontinuum of $K$. The set $K-A$ contains no ample subcontinuum relative to $K$ because $K$ is semi-indecomposable. The continuum $K$ is Kelley, and thus every subcontinuum of $K-A$ is filament relative to $K$. Let $A_{0}$ be a subcontinuum of $K$ containing $A$ in its interior such that $K-A_{0} \neq \emptyset$. There are two points $p_{1}, p_{2} \in K-A_{0}$ such that every continuum in $K$ containing $p_{1}$ and $p_{2}$ intersects $A_{0}$; otherwise, $K$ would contain an ample subcontinuum, $\operatorname{cl}\left(K-A_{0}\right)$, disjoint with $A$, which is impossible.

Since $A_{0}$ is ample in $K$, there is a continuum $B_{0}$ in $K$ containing $A_{0}$ in its interior relative to $K$, such that $p_{1}, p_{2} \notin B_{0}$. Let $C_{1}$ and $C_{2}$ be the components of $K-B_{0}$ containing $p_{1}$ and $p_{2}$, respectively. Then the closures $\mathrm{cl}_{1}$ and $\mathrm{cl}_{2}$ are disjoint continua, both intersecting $B_{0}$, such that $\operatorname{cl}_{1}-B_{0} \neq \emptyset \neq \operatorname{cl} C_{2}-B_{0}$. Hence $Y$ contains a non-semi-terminal subcontinuum, $B_{0}$, a contradiction.

If $K$ is a subcontinuum of a space $X$ such that every subcontinuum of $K$ is terminal in $X$, we say that $K$ is a hereditarily terminal subcontinuum of $X$. The next result follows from Corollary 4.4 and Proposition 4.8 . 
Corollary 4.9. Each hereditarily semi-terminal subcontinuum of a Kelley continuum is hereditarily indecomposable and hereditarily terminal.

The next corollary follows from Proposition 3.10 and Corollary 4.9.

Corollary 4.10. For each Kelley continuum $X$ the following conditions are equivalent:

(a) $X$ is hereditarily indecomposable,

(b) $X$ is hereditarily semi-indecomposable,

(c) each subcontinuum of $X$ is terminal in $X$, and

(d) each subcontinuum of $X$ is semi-terminal in $X$.

We end this section with the following "hereditarily (semi-)terminal decomposition theorem" for Kelley continua. The circle of pseudo-arcs [2] and subsequent generalizations [15] are primary examples of continua in which this decomposition is nontrivial and has nondegenerate members. Nevertheless, each hereditarily indecomposable continuum can be a member of a nontrivial decomposition described in this theorem.

Theorem 4.11. Each Kelley continuum $X$ admits a finest monotone, upper semicontinuous decomposition $\mathcal{H}$ such that the quotient space $X / \mathcal{H}$ has no nondegenerate hereditarily semi-terminal subcontinua. Moreover, the decomposition $\mathcal{H}$ can be defined as the collection of the maximal hereditarily terminal (or equivalently, hereditarily semi-terminal) subcontinua of $X$, and the members of $\mathcal{H}$ are hereditarily indecomposable.

Proof. Since the collection $\mathcal{H S T}(X)$ is closed by Proposition 4.7, it follows by the Kuratowski-Zorn lemma that every point $x$ in $X$ belongs to a maximal member $H_{x}$ of $\mathcal{H S T}(X)$. By Corollary 4.9, the members of $\mathcal{H S T}(X)$ are terminal. Therefore, there is only one such set $H_{x}$ for each $x$, and the collection $\mathcal{H}=\left\{H_{x} \mid x \in X\right\}$ partitions $X$. Moreover, by Proposition 4.7 the decomposition $\mathcal{H}$ is upper semicontinuous. Let $q: X \rightarrow X / \mathcal{H}$ be the quotient map of this decomposition. First, we show that $X / \mathcal{H}$ has no proper, nondegenerate hereditarily semi-terminal subcontinuum.

Indeed, suppose the space $X / \mathcal{H}$ has a proper, nondegenerate, hereditarily semiterminal subcontinuum $Y$. Let $Z=q^{-1}(Y)$, and let $T$ be a subcontinuum of $Z$. We want to show that $T$ is semi-terminal in $X$. If $T$ is contained in a single member $H$ of $\mathcal{H}$, then it is semi-terminal in $X$ by the definition of $\mathcal{H}$. Otherwise, since each $H \in \mathcal{H}$ is terminal, $T$ is the union of some members of $\mathcal{H}$. Thus $q^{-1}(q(T))=T$.

Let $K$ and $L$ be disjoint continua in $X$ with $K \cap T \neq \emptyset \neq L \cap T$. If either $K$ or $L$ is contained in a single member of $\mathcal{H}$, then either $K \subset T$ or $L \subset T$, because $T$ is saturated with respect to $q$.

Assume neither $K$ nor $L$ is contained in a single member of $\mathcal{H}$. Then $q^{-1}(q(K))=$ $K$ and $q^{-1}(q(L))=L$ by the terminality of the members of $\mathcal{H}$. Consequently, $q(K)$ and $q(L)$ are disjoint continua both intersecting $q(T)$. The continuum $q(T)$ is a subcontinuum of $Y$, and thus it is semi-terminal in $X / \mathcal{H}$. Therefore, either $q(K) \subset q(T)$ or $q(L) \subset q(T)$. Since $T$ is saturated with respect to $q$, either $K \subset T$ or $L \subset T$.

Thus $T$ is semi-terminal in $X$. Consequently $Z=q^{-1}(Y)$ is hereditarily semiterminal in $X$ and $Y=q(Z)$ is a singleton by the definition of $q$, a contradiction. We have shown that $X / \mathcal{H}$ has no proper, nondegenerate hereditarily semi-terminal subcontinua. 
Suppose $\mathcal{F}$ is a monotone, upper semi-continuous decomposition of $X$ such that $\mathcal{H}$ is not finer than $\mathcal{F}$, which means that a member $H$ of $\mathcal{H}$ is not contained in a single member of $\mathcal{F}$. Let $f: X \rightarrow X / \mathcal{F}$ be the quotient map of $\mathcal{F}$. Then $f(H)$ is a nondegenerate subcontinuum of $X / \mathcal{F}$, which is hereditarily semi-terminal in $X / \mathcal{F}$ by Proposition 3.4. The proof is complete.

\section{Aposyndetic Decomposition of Kelley continua}

In this section we add some improvements to the aposyndetic decomposition theorem for Kelley continua presented in 23 and show other facts about the structure of Kelley continua related to that decomposition. This theorem generalizes Jones' aposyndetic theorem [11, later improved by Rogers [25], 26, 28, 29].

Given a proper subcontinuum $Y$ of a continuum $X$, the set of all outlet points of $Y$ can be expressed as $\bigcap\{Z \in C(X) \mid Z \cap Y \neq \emptyset \neq Z-Y\}$. Thus the set of outlet points of $Y$ is compact. If $Y=X$, then each point of $Y$ is its outlet point. As is noticed in section 2, every continuum having an outlet point is semi-terminal. Example 2.2 shows that, in general, the converse is not true. An easy proof of the following proposition is left to the reader.

Proposition 5.1. If $X$ is a Kelley continuum, then the collections $\{(K, x) \mid x$ is an outlet point of $K \in C(X)\}$ and $\{K \in C(X) \mid K$ has an outlet point $\}$ are closed in $C(X) \times X$ and $C(X)$, respectively.

The following proposition is a consequence of Proposition 4.2 .

Proposition 5.2. If a subcontinuum $Y$ of a Kelley space $X$ has an outlet point, then $Y$ is a semi-terminal, Kelley subcontinuum of $X$.

If $X$ is a continuum and $x \in X$, define

$$
L(x)=\{y \in X \mid X \text { is not aposyndetic at } y \text { with respect to } x\} .
$$

Directly from this definition we have the following.

Proposition 5.3. If $X$ is a continuum and $x \in X$, then $x \in A$ for every ample subcontinuum $A$ of $X$ intersecting $L(x)$.

The sets $L(x)$ are called $L$-sets. It is known that the $L$-sets are continua [10. If $X$ is a Kelley continuum, we have $L(x)=X-\bigcup\{A \in C(X) \mid A$ is an ample and $x \notin$ $A\}$, and also $x \in L(y)$ if and only if $L(x) \subset L(y)$ [23, Corollary 2.8, p. 5994]. Thus the relation $x \preceq * y$, defined by $x \in L(y)$, is a quasi-order, and it is a closed subset of $X \times X$ [23, Proposition 3.1, p. 5996]. Consequently, in Kelley continua there are maximal and minimal $L$-sets. The following has been proved in 23 , Proposition 4.2, p. 5997].

Proposition 5.4. If $X$ is a Kelley continuum, then $x$ is an outlet point of $L(x)$ for every $x \in X$.

The next proposition follows from the definition.

Proposition 5.5. If $X$ is a continuum, then $L(x)=X$ if and only if $x$ is a cofilament point of $X$. 
Corollary 5.6. If $X$ is a Kelley continuum, then each L-set is a semi-terminal, Kelley continuum, and each minimal L-set is terminal.

Proof. The first conclusion is a consequence of Propositions 5.2 and 5.4 Note that if $L(x)$ is a minimal $L$-set and $y \in L(x)$, then $L(y)=L(x)$. Therefore, each point of $L(x)$ is its outlet point by Proposition [5.3. Hence $L(x)$ is terminal in $X$.

Proposition 5.7. Let $X$ be a Kelley continuum, $Y$ a semi-terminal subcontinuum of $X$, and $x \in Y$. If $L_{Y}(x)$ is the $L$-set in $Y$ determined by $x$, then $x$ is an outlet point of $L_{Y}(x)$ in $X$. In particular, every $L$-set in $Y$ is a semi-terminal subcontinuum of $X$.

Proof. Let $K$ be a subcontinuum of $X$ such that $K \cap L_{Y}(x) \neq \emptyset \neq K-L_{Y}(x)$ and $A=K \cap Y$. If $K \subset Y$, then $x \in K$, because $x$ is an outlet point of $L_{Y}(x)$ in $Y$ (Proposition [5.4). If $K-Y \neq \emptyset$, then, by Proposition 4.3, $A$ is an ample subcontinuum of $Y$ intersecting $L_{Y}(x)$. Thus $x \in A \subset K$ by Proposition 5.3.

Let $\mathcal{G}=\mathcal{G}(X)$ be the collection of all maximal $L$-sets in a continuum $X$, i.e.

$$
\mathcal{G}=\left\{L(x) \mid x \text { is maximal in } X \text { with respect to } \preceq^{*}\right\} .
$$

In 23 a generalization of Jones' aposyndetic decomposition theorem for Kelley continua was shown. In that result, the collection $\mathcal{G}(X)$ is the generalized aposyndetic decomposition of a Kelley continuum $X$. Here we present an improved version of this theorem. The new addition is that the fibers of the decomposition are semi-terminal, Kelley subcontinua of the space.

Theorem 5.8 (Aposyndetic Decomposition Theorem for Kelley continua). If $X$ is a Kelley continuum, then $\mathcal{G}(X)$ is an upper semi-continuous decomposition of $X$ into semi-terminal, Kelley continua, and the quotient space $X / \mathcal{G}(X)$ is an aposyndetic Kelley continuum.

Moreover, $\mathcal{G}(X)$ is the finest monotone, upper semi-continuous decomposition of $X$ such that the quotient space is aposyndetic. That is, for every monotone, upper semi-continuous decomposition $\mathcal{D}$ of $X$ with an aposyndetic quotient, each member of $\mathcal{G}(X)$ is a subset of some member of $\mathcal{D}$.

The next corollary follows from Proposition 5.5 and Theorem 5.8

Corollary 5.9. If $X$ is a Kelley continuum, then $\mathcal{G}(X)$ is trivial (that is, it has only one member, $X)$ if and only if $X$ has a cofilament point.

Corollary 5.10. If $X$ is a Kelley continuum and $\mathcal{G}(X)$ is trivial, then $X$ is semiindecomposable.

Remark 5.11. The converse to Corollary 5.10 in general is not true even among homogeneous continua. Indeed, if $X$ is the product of two pseudo-arcs, then $X$ is aposyndetic. Therefore, its aposyndetic decomposition is composed only of singletons. Nevertheless, this space is known to be semi-indecomposable 9 , Theorem 10, p. 621]. 
Proposition 5.12. Let $Y$ be a semi-terminal subcontinuum of a Kelley continuum $X$. If $X$ is aposyndetic at $x$ with respect to $y$ and $x, y \in Y$, then $Y$ is aposyndetic at $x$ with respect to $y$. Consequently, if $X$ is aposyndetic, then so is $Y$.

Proof. Let $P$ be a continuum in $X$ such that $x \in \operatorname{Int} P$ and $y \notin P$. Then $P \cap Y$ is a continuum by Proposition 3.1, it contains $x$ in its interior relative to $Y$, and $y \notin P \cap Y$.

Note that if $Y$ is a semi-terminal subcontinuum of a Kelley continuum $X$, then, by Corollary 3.3, the collection $\{D \cap Y \mid D \in \mathcal{G}(X)\}$ is a monotone, upper semicontinuous decomposition of $Y$.

Proposition 5.13. Let $Y$ be a semi-terminal subcontinuum of a Kelley continuum $X$. Then the aposyndetic decomposition $\mathcal{G}(Y)$ of $Y$ is finer than the decomposition $\mathcal{F}=\{D \cap Y \mid D \in \mathcal{G}(X)\}$.

Proof. Let $q: X \rightarrow X / \mathcal{G}(X)$ be the quotient map of $\mathcal{G}(X)$. By Corollary 3.3 the restriction $q \mid Y: Y \rightarrow q(Y)$ is also monotone. The continuum $q(X)=X / \mathcal{G}(X)$ is aposyndetic and Kelley by Theorem [5.8 and $q(Y)$ is its semi-terminal subcontinuum by Proposition 3.4. Therefore, $q(Y)$ is aposyndetic by Proposition 5.12, The continuum $Y / \mathcal{F}$ is homeomorphic to $q(Y)$, and thus it is aposyndetic. Hence $\mathcal{G}(Y)$ is finer than $\mathcal{F}$ by Theorem 5.8 .

If $X$ is a continuum, let $\operatorname{Cfm}(X)$ be the set of cofilament points of $X$. For any topological space $X$, let $\widehat{\mathcal{A}}(X)$ be the collection of all semi-terminal continua $Y$ in $X$ such that $\operatorname{Cfm}(Y) \neq \emptyset$. Recall that for a Kelley continuum $X$ and $Y \in$ $\widehat{\mathcal{A}}(X)$, the defining condition $\operatorname{Cfm}(Y) \neq \emptyset$ is essentially stronger than the semiindecomposability of $Y$, and it is equivalent to having the intersection of ample subcontinua of $Y$ nonempty. Moreover, in such $X$ each member of $\widehat{\mathcal{A}}(X)$ has an outlet point by Corollary 4.4.

Proposition 5.14. Let $X$ be a space and $Y$ a member of $\widehat{\mathcal{A}}(X)$. For every Kelley continuum $Z$ in $X$ such that $Y \subset Z$, the continuum $Y$ is contained in a single member of the aposyndetic decomposition $\mathcal{G}(Z)$ of $Z$.

Proof. Observe that $Y \in \widehat{\mathcal{A}}(Z)$. By Corollary [5.9, the decomposition $\mathcal{G}(Y)$ is trivial, and it is finer than $\{Y \cap D \mid D \in \mathcal{G}(Z)\}$ by Proposition 5.13. The conclusion follows.

In the proofs of the next two propositions we use the following observation. Let $\mathcal{A}(X)$ be the collection of ample continua in a Kelley continuum $X$, and assume $\operatorname{Cfm}(X)=\emptyset$. Since $\operatorname{Cfm}(X)=\bigcap \mathcal{A}(X)$, we have $\bigcap \mathcal{A}(X)=\emptyset$, and thus $\bigcup\{X-$ $A \mid A \in \mathcal{A}(X)\}=X$. Since $X$ is compact, $X=\left(X-A_{1}\right) \cup \cdots \cup\left(X-A_{n}\right)$, or equivalently $A_{1} \cap \cdots \cap A_{n}=\emptyset$, for some finite collection $\left\{A_{1}, \ldots, A_{n}\right\}$ of ample subcontinua of $X$.

Proposition 5.15. If $X$ is a Kelley continuum, $Y, Z \in \widehat{\mathcal{A}}(X)$, and $Y \cap Z \neq \emptyset$, then $Y \cup Z \in \widehat{\mathcal{A}}(X)$.

Proof. If either $Y \subset Z$ or $Z \subset Y$, the conclusion is trivial. Assume $Y-Z \neq \emptyset \neq$ $Z-Y$. By Proposition 3.6. $Y \cup Z$ is semi-terminal in $X$.

Suppose $\operatorname{Cfm}(Y \cup Z)=\emptyset$. Then $A_{1} \cap \cdots \cap A_{n}=\emptyset$ for some ample subcontinua $A_{1}, \ldots, A_{n}$ of $Y \cup Z$. Note that $A_{j} \cap Y \neq \emptyset$ for each $j \in\{1, \ldots, n\}$. Indeed, otherwise 
$A_{j} \subset Z-Y$, and $A_{j}$ is also ample in $Z$. The set $Y \cap Z$ is an ample subcontinuum of $Z$ by Proposition 4.3, which is disjoint with $A_{j}$. Thus $\operatorname{Cfm}(Z)=\emptyset$, contrary to the assumption.

Since $A_{1}, \ldots, A_{n}$ are ample continua in $Y \cup Z$ and $Y$ is semi-terminal in $Y \cup Z$, by Corollary 3.2 it follows that $A_{1} \cap Y, \ldots, A_{n} \cap Y$ are ample subcontinua of $Y$. Consequently, $\emptyset \neq \operatorname{Cfm}(Y) \subset\left(A_{1} \cap Y\right) \cap \cdots \cap\left(A_{n} \cap Y\right) \subset A_{1} \cap \cdots \cap A_{n}$, a contradiction.

Proposition 5.16. If $X$ is a Kelley continuum and $p \in X$, there exists a largest member of $\widehat{\mathcal{A}}(X)$ containing $p$.

Proof. Let $\widehat{\mathcal{A}}(X, p)=\{K \in \widehat{\mathcal{A}}(X) \mid p \in K\}$, and note that $\{p\} \in \widehat{\mathcal{A}}(X, p)$. Thus $\widehat{\mathcal{A}}(X, p) \neq \emptyset$. By Proposition 5.15, it suffices to show that every member of $\widehat{\mathcal{A}}(X, p)$ is contained in a maximal member of $\widehat{\mathcal{A}}(X, p)$. We apply the Kuratowski-Zorn lemma to show this.

Let $M_{\xi}$ be a collection of members of $\widehat{\mathcal{A}}(X, p)$, where $\xi$ is in a set $\Xi$ strictly ordered by a relation $\preceq$, and $M_{\xi_{1}} \subset M_{\xi_{2}}$ whenever $\xi_{1} \preceq \xi_{2}$. Since the sets $M_{\xi}$ are closed subsets of a compact space, and they are strictly ordered by inclusion, it follows that $\bigcup\left\{M_{\xi} \mid \xi \in \Xi\right\}=\bigcup\left\{M_{n} \mid n \in\{1,2, \ldots\}\right\}$ for some sets $M_{n}$ selected from the collection $\left\{M_{\xi} \mid \xi \in \Xi\right\}$ and satisfying $M_{n} \subset M_{n+1}$ for each $n$. The continuum $M_{0}=\operatorname{Lim} M_{n}$ is an upper bound of the collection $\left\{M_{\xi} \mid \xi \in \Xi\right\}$. To use the Kuratowski-Zorn lemma as desired, it suffices to show that $M_{0} \in \widehat{\mathcal{A}}(X, p)$.

Clearly, $p \in M_{0}$. Being the limit of the semi-terminal continua $M_{n}$, the continuum $M_{0}$ is semi-terminal by Proposition 4.5, To complete the proof it suffices to show that $\operatorname{Cfm}\left(M_{0}\right) \neq \emptyset$.

Suppose $\operatorname{Cfm}\left(M_{0}\right)=\emptyset$. Then $A_{1} \cap \cdots \cap A_{m}=\emptyset$ for some ample subcontinua $A_{1}, \ldots, A_{m}$ of $M_{0}$. These continua can be slightly enlarged to the corresponding continua $B_{1}, \ldots, B_{m}$ such that for each $j$ the set $A_{j}$ is in the interior of $B_{j}$ relative to $M_{0}$ and $B_{1} \cap \cdots \cap B_{m}=\emptyset$. Since the relative interior of $B_{j}$ in $M_{0}$ is nonempty, $B_{j} \cap M_{n} \neq \emptyset$ for almost all $n$. Thus $B_{j} \cap M_{n} \neq \emptyset$ for all $j \in\{1, \ldots, m\}$, and $n$ is greater than some number $C$. Fix an $n>C$. By Corollary 3.2, the set $B_{j} \cap M_{n}$ is an ample subcontinuum of $M_{n}$ for each $j$. Since $M_{n} \in \widehat{\mathcal{A}}(X, p) \subset \widehat{\mathcal{A}}(X)$, it follows that $\emptyset \neq\left(B_{1} \cap M_{n}\right) \cap \cdots \cap\left(B_{m} \cap M_{n}\right) \subset B_{1} \cap \cdots \cap B_{m}$, a contradiction. The proof is complete.

By Propositions 5.15 and 5.16, the maximal members of $\widehat{\mathcal{A}}(X)$ form a unique partition of a Kelley continuum $X$. Let $\Gamma(X)$ be that partition, and let $\Gamma(X, x)$ be the member of $\Gamma(X)$ containing a point $x \in X$. Similarly, let $\mathcal{G}(X, x)$ be the member of the aposyndetic decomposition $\mathcal{G}(X)$ of $X$ containing $x \in X$. By Proposition 5.14 we have the following.

Corollary 5.17. If $X$ is a Kelley continuum, then the partition $\Gamma(X)$ is finer than the aposyndetic decomposition $\mathcal{G}(X)$; that is, $\Gamma(X, x) \subset \mathcal{G}(X, x)$ for each $x \in X$.

By the Aposyndetic Decomposition Theorem, Theorem 5.8, the sets $\mathcal{G}(X, x)$ are Kelley continua if $X$ is Kelley. Thus we can apply this theorem to each $\mathcal{G}(X, x)$ and obtain the aposyndetic decomposition $\mathcal{G}(\mathcal{G}(X, x))$. A semi-terminal subcontinuum of a semi-terminal continuum does not have to be semi-terminal in the whole space. There are counterexamples, even in $X=[0,1]$. Nevertheless, by Proposition 5.7 the members of $\mathcal{G}(\mathcal{G}(X, x))$ are semi-terminal, not only in $\mathcal{G}(X, x)$ but also in $X$. 
Given a Kelley continuum $X$ and $x \in X$, let $\mathcal{G}_{0}(X, x)=X$. Suppose for some ordinal $\alpha$ a partition of $X$ is defined into semi-terminal, Kelley subcontinua $\mathcal{G}_{\alpha}(X, x)$, where $\mathcal{G}_{\alpha}(X, x)$ is the member of that partition containing $x$. Then we let $\mathcal{G}_{\alpha+1}(X, x)=\mathcal{G}\left(\mathcal{G}_{\alpha}(X, x), x\right)$. By Proposition 5.7 it follows that $\mathcal{G}_{\alpha+1}(X, x)$ is semi-terminal. Therefore, $\mathcal{G}_{\alpha+1}(X, x)$ is a Kelley continuum by Proposition 4.2 . For a limit ordinal $\beta \neq 0$ define $\mathcal{G}_{\beta}(X, x)=\bigcap\left\{\mathcal{G}_{\alpha}(X, x) \mid \alpha<\beta\right\}$. Note that such $\mathcal{G}_{\beta}(X, x)$ is the limit of the sets $\mathcal{G}_{\alpha}(X, x)$ for $\alpha<\beta$. If all $\mathcal{G}_{\alpha}(X, x)$ 's are semiterminal in $X$, then so is $\mathcal{G}_{\beta}(X, x)$ by Proposition 4.5. Consequently, $\mathcal{G}_{\beta}(X, x)$ is a Kelley continuum by Proposition 4.2. By induction, a nested, well-ordered sequence of Kelley, semi-terminal continua $\mathcal{G}_{\alpha}(X, x)$ is associated with each $x \in X$. For each $\alpha$ the collection $\mathcal{G}_{\alpha}(X)=\left\{\mathcal{G}_{\alpha}(X, x) \mid x \in X\right\}$ is a partition of $X$. Using Propositions 5.1 and 5.7, we also observe that for every $\alpha$ and $x \in X$ the set $\mathcal{G}_{\alpha}(X, x)$ is a continuum having an outlet point. Since $X$ is a metric compactum, for every $x \in X$ there is the smallest countable ordinal $\gamma$ such that $\mathcal{G}_{\gamma}(X, x)=\mathcal{G}_{\gamma+1}(X, x)$. Let $\gamma(x)=\gamma(X, x)$ be that ordinal. We note that $\left\{\mathcal{G}_{\gamma(x)}(X, x) \mid x \in X\right\}$ is a partition of $X$ into semi-terminal continua.

Proposition 5.18. The partition $\left\{\mathcal{G}_{\gamma(x)}(X, x) \mid x \in X\right\}$ is identical with the partition $\Gamma(X)$ of $X$ into the maximal members of $\widehat{\mathcal{A}}(X)$.

Proof. Let $x \in X$. Since $\mathcal{G}_{\gamma(x)}(X, x)=\mathcal{G}_{\gamma(x)+1}(X, x)$, the aposyndetic decomposition of $\mathcal{G}_{\gamma(x)}(X, x)$ is trivial, and thus $\mathcal{G}_{\gamma(x)}(X, x)$ has a cofilament point by Corollary [5.9, The continuum $\mathcal{G}_{\gamma(x)}(X, x)$ is semi-terminal in $X$. Consequently $\mathcal{G}_{\gamma(x)}(X, x) \in \widehat{\mathcal{A}}(X)$. Since $\Gamma(X, x)$ is the largest member of $\widehat{\mathcal{A}}(X)$ containing $x$, it follows that $\mathcal{G}_{\gamma(x)}(X, x) \subset \Gamma(X, x)$.

The continuum $\Gamma(X, x)$ has an outlet point with respect to the space $X$. Therefore it has an outlet point with respect to each continuum $Y \subset X$ containing $\Gamma(X, x)$. Thus, for any such $Y$, the continuum $\Gamma(X, x)$ is semi-terminal in $Y$, and $\Gamma(X, x) \in \widehat{\mathcal{A}}(Y)$. By Proposition 5.14 we have $\Gamma(X, x) \subset \mathcal{G}(X, x)=\mathcal{G}_{1}(X, x)$. More generally, using Proposition 5.14 we conclude that $\Gamma(X, x) \subset \mathcal{G}_{\alpha+1}(X, x)$ whenever $\Gamma(X, x) \subset \mathcal{G}_{\alpha}(X, x)$. By induction, $\Gamma(X, x) \subset \mathcal{G}_{\alpha}(X, x)$ for every $\alpha$. In particular $\Gamma(X, x) \subset \mathcal{G}_{\gamma(x)}(X, x)$. The proof is complete.

\section{Four decompositions of Kelley continua}

If $\mathcal{P}_{1}$ and $\mathcal{P}_{2}$ are partitions of a set $S$, we write $\mathcal{P}_{1} \sqsubseteq \mathcal{P}_{2}$ whenever $\mathcal{P}_{1}$ is finer than $\mathcal{P}_{2}$; that is, each member of $\mathcal{P}_{1}$ is contained in some member of $\mathcal{P}_{2}$. The following three partitions of a Kelley continuum $X$ into semi-terminal subcontinua are discussed in sections 4 and 5 .

(i) the partition $\mathcal{H}(X)$ into the maximal hereditarily terminal subcontinua of $X$,

(ii) the partition $\Gamma(X)$ into the maximal semi-terminal subcontinua of $X$ having a cofilament point, and

(iii) the partition $\mathcal{G}(X)$ into the maximal $L$-sets in $X$.

Each member of the partitions $\mathcal{H}(X), \Gamma(X)$ and $\mathcal{G}(X)$ has an outlet point, and both $\mathcal{H}(X)$ and $\mathcal{G}(X)$ are upper semi-continuous decompositions. The partition $\Gamma(X)$, in general, is neither upper nor lower semi-continuous. These partitions satisfy the relations $\mathcal{H}(X) \sqsubseteq \Gamma(X) \sqsubseteq \mathcal{G}(X)$. 
Remark 6.1. There exists a finest upper semi-continuous decomposition $\Gamma_{0}(X)$ of a Kelley continuum $X$ into closed sets such that $\Gamma(X)$ is finer than $\Gamma_{0}(X)$. The members of $\Gamma_{0}(X)$ are also semi-terminal subcontinua of $X$ with outlet points. Clearly $\Gamma(X) \sqsubseteq \Gamma_{0}(X) \sqsubseteq \mathcal{G}(X)$. The decomposition $\Gamma_{0}(X)$ is not in the focus of this paper, though it may be an interesting subject for future study.

The fourth partition from the title of this section is the mutually aposyndetic decomposition $\mathcal{Q}(X)$ of a Kelley continuum $X$ defined in [18. The decomposition $\mathcal{Q}(X)$ is upper semi-continuous, and its members are closed. It is defined by the following equivalence relation: If $x, y \in X$, then $x$ is equivalent to $y$ if and only if every ample continua $A_{x}$ and $A_{y}$ in $X$ containing $x$ and $y$, respectively, have nonempty intersection. Observe that each $L$-set in $X$ is contained in a single member of the decomposition $\mathcal{Q}(X)$ by the definition of $\mathcal{Q}(X)$. Thus $\mathcal{G}(X) \sqsubseteq \mathcal{Q}(X)$. As it is noted in Remark 2.3. the decomposition of the continuum $\hat{X}_{\Delta}^{2}$ defined in Example 2.2, into the continua $(q \times q)^{-1}(p)$, equals $\mathcal{Q}\left(\hat{X}_{\Delta}^{2}\right)$ whenever $X$ is a mutually aposyndetic, homogeneous curve. The members of this decomposition are semi-terminal continua without outlet points. Note that these members are products of two pseudo-arcs, and thus they are semi-indecomposable continua without cofilament points.

For a Kelley continuum $X$ and an ordinal $\alpha>0$, let $\mathcal{G}_{\alpha}(X)$ be the partition of $X$ defined in the previous section. The partitions $\mathcal{G}_{\alpha}(X)$, for $\alpha>1$, and $\Gamma(X)$ are neither upper nor lower semi-continuous. If $\alpha_{2} \geq \alpha_{1}>0$, we have the following:

$$
\mathcal{H}(X) \sqsubseteq \Gamma(X) \sqsubseteq \mathcal{G}_{\alpha_{2}}(X) \sqsubseteq \mathcal{G}_{\alpha_{1}}(X) \sqsubseteq \mathcal{G}_{1}(X)=\mathcal{G}(X) \sqsubseteq \mathcal{Q}(X) .
$$

If $X$ is a homogeneous continuum, it follows from Jones' aposyndetic theorem [11] that $\gamma(x)=1$ for every $x \in X$, and $\mathcal{G}(X)=\mathcal{G}_{1}(X)=\Gamma(X)$ is the aposyndetic decomposition of $X$. For each of the decompositions $\mathcal{H}(X), \mathcal{G}(X)$ and $\mathcal{Q}(X)$ we have numerous examples of homogeneous continua $X$ such that the decomposition is nontrivial and has nondegenerate members. We do not know whether $\mathcal{H}(X)=$ $\mathcal{G}(X)$ for every decomposable homogeneous continuum $X$. By the main results of [13] and [27] this last problem is equivalent to [28, Question 1, p. 377].

Question 2. Does there exist a homogeneous continuum $X$ such that at least two of the decompositions $\mathcal{H}(X), \mathcal{G}(X)$ and $\mathcal{Q}(X)$ : (i) are different; (ii) are nontrivial; and (iii) have nondegenerate members?

The following question, which was originally asked in [18, Question 2], is related to Question 1 from section 2

Question 3. Is every aposyndetic, homogeneous curve mutually aposyndetic?

In the general case, the following two fundamental questions concerning the mutually aposyndetic decomposition $\mathcal{Q}(X)$ remain open (compare [18, Question 1]).

Question 4. If $X$ is a Kelley continuum, is the quotient $X / \mathcal{Q}(X)$ a Kelley continuum?

Question 5. If $X$ is a Kelley continuum, is each member of $\mathcal{Q}(X)$ connected? If yes, is it a semi-indecomposable continuum? 


\section{ACKNOWLEDGMENT}

The author thanks the referee for his/her suggestions which helped to simplify some proofs and eliminate some mistakes and inconsistencies in this article.

\section{REFERENCES}

[1] R. H. Bing, Higher-dimensional hereditarily indecomposable continua, Trans. Amer. Math. Soc. 71 (1951), 267-273. MR0043452 (13:265c)

[2] R. H. Bing and F. B. Jones, Another homogeneous plane continuum, Trans. Amer. Math. Soc. 90 (1959), 171-192. MR0100823 (20:7251)

[3] J. J. Charatonik, W. J. Charatonik and J. R. Prajs, Kernels of hereditarily unicoherent continua and absolute retracts, Topology Proc. 26 (2001/02), 127-145. MR1966987 (2004b:54053)

[4] - Arc property of Kelley and absolute retracts for hereditarily unicoherent continua, Colloq. Math. 97 (2003), 49-65. MR2010542 (2004h:54028)

[5] J. J. Charatonik, W. J. Charatonik and J. R. Prajs, Hereditarily unicoherent continua and their absolute retracts, Rocky Mountain J. Math. 34 (2004), 83-110. MR2061119 (2005b:54058)

[6] , Atriodic absolute retracts for hereditarily unicoherent continua, Houston J. Math. 30 (2004), 1069-1087. MR2110250 (2005j:54022)

[7] J. J. Charatonik and J. R. Prajs, AANR spaces and absolute retracts for tree-like continua, Czechoslovak Math. J. 55 (2005), 877-891. MR2184369 (2006h:54012)

[8] W. J. Charatonik, The Lelek fan is unique, Houston J. Math. 15 (1989), 27-34. MR.1002079 (90f:54050)

[9] C. L. Hagopian, Mutual aposyndesis, Proc. Amer. Math. Soc. 23 (1969), 615-622. MR0247612(40:876)

[10] F. B. Jones, Concerning non-aposyndetic continua, Amer. J. Math. 70 (1948), 403-413. $\operatorname{MR} 0025161(9: 606 \mathrm{~h})$

[11] , On a certain type of homogeneous plane continuum, Proc. Amer. Math. Soc. 6 (1955), 735-740. MR0071761 (17:180e)

[12] J. L. Kelley, Hyperspaces of a continuum, Trans. Amer. Math. Soc. 52 (1942), 22-36. MR0006505 (3:315b)

[13] P. Krupski and J. R. Prajs, Outlet points and homogeneous continua, Trans. Amer. Math. Soc. 318 (1990), 123-141. MR937246 (90f:54054)

[14] A. Lelek, On plane dendroids and their end points in the classical sense, Fund. Math. 49 (1960/1961), 301-319. MR0133806 (24:A3631)

[15] W. Lewis, Continuous curves of pseudo-arcs, Houston J. Math. 11 (1985), 91-99. MR.780823 (86e:54038)

[16] S. B. Nadler, Jr., Continuum theory, Marcel Dekker, Inc., New York, 1992. MR.1192552 (93m:54002)

[17] J. R. Prajs, A homogeneous arcwise connected non-locally-connected curve, Amer. J. Math. 124 (2002), 649-675. MR 1914455 (2003f:54077)

[18] _ Mutually aposyndetic decomposition of homogeneous continua, Canadian J. Math. 62 (2010), 182-201. MR2597029

[19] _ Mutual aposyndesis and products of solenoids, Topology Proc. 32 (2008), 339-349. MR.1500093

[20] , Co-filament continua in homogeneous spaces, preprint.

[21] J. R. Prajs and K. Whittington, Filament sets and homogeneous continua, Topology Appl. 154 (2007), 1581-1591. MR2317064 (2008e:54032)

[22] , Filament sets and decompositions of homogeneous continua, Topology Appl. 154 (2007), 1942-1950. MR2319265(2008d:54021)

[23] _ Filament sets, aposyndesis, and the decomposition theorem of Jones, Trans. Amer. Math. Soc. 359 (2007), 5991-6000. MR2336313 (2008g:54047)

[24] _ Filament additive homogeneous continua, Indiana Univ. Math. J. 56 (2007), 263277. MR 2305937 (2008g:54046)

[25] J. T. Rogers, Jr., Completely regular mappings and homogeneous, aposyndetic continua, Canad. J. Math. 33 (1981), 450-453. MR617635 (83a:54012) 
[26] , Decompositions of homogeneous continua, Pacific J. Math. 99 (1982), 137-144. MR651491 (83c:54045)

[27] Homogeneous hereditarily indecomposable continua are tree-like, Houston J. Math. 8 (1982), 421-428. MR684167 (84b:54072)

[28] _ Cell-like decompositions of homogeneous continua, Proc. Amer. Math. Soc. 87 (1983), 375-377. MR681852 (84e:54040)

[29] - Higher dimensional aposyndetic decompositions, Proc. Amer. Math. Soc. 131 (2003), 3285-3288. MR 1992870 (2004e:54029)

[30] R. W. Wardle, On a property of J. L. Kelley, Houston J. Math. 3 (1977), 291-299. MR $0458379(56: 16582)$

Department of Mathematics and Statistics, California State University Sacramento, 6000 J Street, Sacramento, California 95819 - and - Institute of Mathematics, UniverSity of Opole, Ul. Oleska 48, 45-052 Opole, Poland

E-mail address: prajs@csus.edu 